\title{
UV Detection on Artificial Uric Acid Using UV-Vis Spectrometer
}

\section{Norazmi N*, Abdul Rasat ZR, Mohamad M and Manap H}

Faculty of Engineering Technology, University Malaysia Pahang, Pahang, Malaysia

\begin{abstract}
The aim of this research work is to measure the concentration and absorption cross-section of artificial uric acid in the Ultraviolet (UV) region using UV-Vis spectrometer. The uric acid sample comes in powder form which has to be dissolved with distilled water to convert it into liquid form. Therefore, it can be placed in the cuvette for the analysis purposes. This research study was proposed to make a comparison with the previous research studies that uric acid was normally extracted from human serum as a real sample. This research study was carried out using an artificial sample of uric acid with the suspended or grits of uric acid which were not totally dissolved. These grits might be artificially assumed as crystallites which is common with Gout disease. Based on the medical perspective, crystallites normally inhibit the human joints which may cause intense pain to the human bones or tissues. In the experiment, the distilled water was used a background or reference spectrum which can be stored and automatically deducted using the Spectra suite software application. Thus, Spectra suite only measures the pure concentration and UV absorption wavelength of the uric acid through the use of the spectrometer. The absorbance data was extracted and substituted into Beer's Lambert Law formula to calculate the value of absorption cross-section. The result shows that the value of UV absorption wavelength and absorption cross-section is really close as reported in the previous research studies. It proves that even the artificial sample of uric acid with the grits still can give a very close result. The UV absorption of uric acid was obtained at $293.99 \mathrm{~nm}$ by four different concentrations. The response time was successfully done in 3 seconds. The resulting curves have noise signals which can be analyzed and reduced using an averaging method to make the curve look sharper and lack of noise.
\end{abstract}

Keywords: Optical sensor; Ultraviolet (UV) absorption UV-Vis spectrometer; Averaging method

\section{Introduction}

Gout is a common disease due to the abnormal level of uric acid in the human body to cause existence of crystallites which inhibit the human joints. It is also known as a type of arthritis or a uric acid disturbance in the human body which give a painful effect to the human joint bones. It may even lead to the other types of disease such as Hyperurecemia when the level of uric acid is too high. Besides that, kidney stone may exist through this disease which may cause renal failure [1]. Uric acid is originally broken down from purine by human body metabolism. Originally, purine is a common compound in certain food such as seafood and red meat. Therefore, the risk of this disease also relies on the daily intake issue. The more purine in the human body, the higher level of uric acid [2]. The crystallites are uneasy to be dissolved in the human blood and they may cause intense pain and swelling to the human joints besides slowing down the body movements [3]. Based on the medical research perspective, uric acid concentration is a 'fingerprinting' of the risk of this disease to happen [4]. By using an optical sensor, the uric acid compound can be detected in the UV wavelength region. It was reported that uric acid was detected and classified at the UV wavelength peak, $292 \mathrm{~nm}$ while the other types of compound were detected at a different wavelength peak. The classification is made based on the UV wavelength absorption. Basically, the UV absorption of the compound is based on their molecular structure and special chemical characteristics towards the UV light radiation [5]. Many techniques have been implemented for the detection of uric acid. Nevertheless, most of the techniques consume a lot of times especially for the analysis purposes. Besides that, certain techniques also require complicated structures to generate their functions. In this research project, UV-Vis spectrometer has been proposed as an optical fiber sensing system which is very effective due to its simplicity, cost effectiveness and fast time response [6]. The working principle is based on the UV light absorption and transmission. This process is relative to the electronic transition where the electromagnetic spectrum energy is required for the movement of an electron from one quantum level to the other level. The energy condition is satisfied by specific wavelength photons and the energy levels also depend on the chemical bonding of the compound [7].

In this research experiment, optical fiber sensor called UV-Vis spectrometer was proposed due to its effectiveness. It is a miniature optical sensor which is more effective on the UV absorption since miniaturization may enhance the efficiencies and reduce the analysis times besides the power consumption [8]. In this research, the UVVis spectrometer was examined to measure the concentration and UV wavelength absorption. The value of absorption cross-section was calculated by utilizing the Beer's Lambert Law formula. Four samples with different concentrations were prepared. Starting with the uric acid stock, it was serially diluted to the other three concentrations based on the molarity formula calculations. The samples still contained the grits or suspended particles of uric acid which were not totally dissolved in the distilled water. This research experiment was proposed to examine whether the result shows a big or small different to the values which were reported in the previous research studies. The result shows that the UV wavelength peak of uric acid was acquired at $293.99 \mathrm{~nm}$ which is discretely close to $292 \mathrm{~nm}$ and $293 \mathrm{~nm}$. Advantageously, the suspended particles of uric acid can be assumed as artificial crystallites to imitate the real ones formed by the Gout disease. The noise signal on

*Corresponding author: Norazmi N, Faculty of Engineering Technology University Malaysia Pahang, Lebuhraya Tun Razak, 26300 Gambang, Kuantan, Pahang, Malaysia, Tel: +60 9-424 5000; E-mail: mazlee.greenfinite@gmail.com

Received February 18, 2018; Accepted March 12, 2018; Published March 19 2018

Citation: Norazmi N, Abdul Rasat ZR, Mohamad M, Manap H (2018) UV Detection on Artificial Uric Acid Using UV-Vis Spectrometer. J Laser Opt Photonics 5: 179 doi: 10.4172/2469-410X.1000179

Copyright: (c) 2018 Norazmi N, et al. This is an open-access article distributed under the terms of the Creative Commons Attribution License, which permits unrestricted use, distribution, and reproduction in any medium, provided the original author and source are credited. 
the resulting curve might be caused by the turbidity of the suspended particles or grits which affects the measurement. Basically, turbidity causes a cloudy appearance on the sample due to the existence of the suspended particles. It also relates to the optical properties of the liquid which causes the light rays to get scattered and absorbed instead of transmitting in a straight light beam through the sample [9].

Since the resulting curve has noise signals, it can be significantly reduced using an average method. Comparative analysis can be done on the resulting signals by using different averaging points [10]. In this research experiment, the absorbance points of three different wavelength peaks were averaged by three-point interval to produce a smoother curve signal. Three-point interval was chosen since it may give a required result. The new signal looks sharp with less noise peaks. This proves that the potential of UV-Vis spectrometer to examine the uric acid with the existence of uric acid grits in the UV wavelength region is still high. Either by an artificial sample or a real sample of uric acid, they still can give a very close result.

\section{Material and Methods}

\section{Materials}

Instrumentation: A UV-VIS Ocean Optics Maya 2000 Pro spectrometer is set up to UV wavelength region using Deuterium light source. A plastic cuvette (10 mm path length) is used for placing uric acid samples for the analysis phase.

Reagent: Uric acid stock $(1.0 \mathrm{mg} / \mathrm{ml})$ is prepared by dissolving 100 $\mathrm{mg}$ of uric acid powder in $15 \mathrm{~mL}$ of distilled water. This mixture is heated up to $60^{\circ} \mathrm{C}$ on a hot plate and stirred using a magnetic stirrer on the hot plate. It is cooled down to a comfort room temperature, $20-26^{\circ} \mathrm{C}$. $85 \mathrm{~mL}$ of distilled water is added to the mixture up to $100 \mathrm{~mL}$ in order to prepare a uric acid stock. This uric acid stock can be refrigerated for one week and can be repetitiously used for analysis purposes. It can be diluted into three different concentrations; $0.8 \mathrm{mg} / \mathrm{ml}, 0.6 \mathrm{mg} / \mathrm{ml}$ and $0.4 \mathrm{mg} / \mathrm{mL}$ based on the molarity formula calculations.

Experimental procedure: Uric acid sample is placed in a $10-\mathrm{mm}$ path length cuvette. Cuvette is a cell to place the experimental sample which is supposed be in liquid form. Figure 1 shows a complete experimental setup which is used for the detection of uric acid. The spectrometer is connected to CPU (installed with Spectrasuite software) using a USB cable. The optical fiber cables are connected from the Deuterium light source to inner port of the cuvette holder and the outer port of the cuvette holder to the spectrometer Maya 2000 Pro Ocean Optics. Before starting the experiment, warm the Deuterium light source up to 20 seconds. The integration time is set till there is no saturation displayed on the graph of intensity versus intended wavelength region, $200 \mathrm{~nm}-400 \mathrm{~nm}$. Every sample has to be tested and analyzed separately (Figure 1).

Beer's Lambert Law formula and calculations: The calculation of the absorption and concentration of uric acid is based on Beer's Lambert Law formula as shown in the equation 1.0. The values of transmitting and incident light intensity can be substituted into this equation. The standard equation can be utilized and derived to an equation 1.1 that the value of absorption-cross section can be directly calculated.

$$
\begin{aligned}
& A=\varepsilon c l \\
& A=\log _{10}\left(I_{o} / I_{T}\right) \\
& \sigma=\frac{\log _{10}\left(I_{o} / I_{T}\right) \times 2.303}{N \times b} \\
& A=\text { absorbance }
\end{aligned}
$$

$\varepsilon=$ molar absoptivity, a constant as a specific value belongs to chemical characteristics of the experimental sample

$$
\mathrm{c}=\text { concentration }
$$

lor $b=$ path length

Io=Incident light intensity

IT=Transmitting light intensity

$\mathrm{N}=$ concentration in molecules $/ \mathrm{cm}^{3}$ unit.

From the calculations using the equation, it can indicate that a same or a very close value of absorption cross-section of uric acid can be obtained even by different concentrations. This is due the molecular structure of uric acid which is special to give its effects to the UV absorption. Meanwhile, the concentration of uric acid is based on the amount of uric acid in each sample.

\section{Result and Discussion}

The analysis of uric acid detection was done by investigating the UV

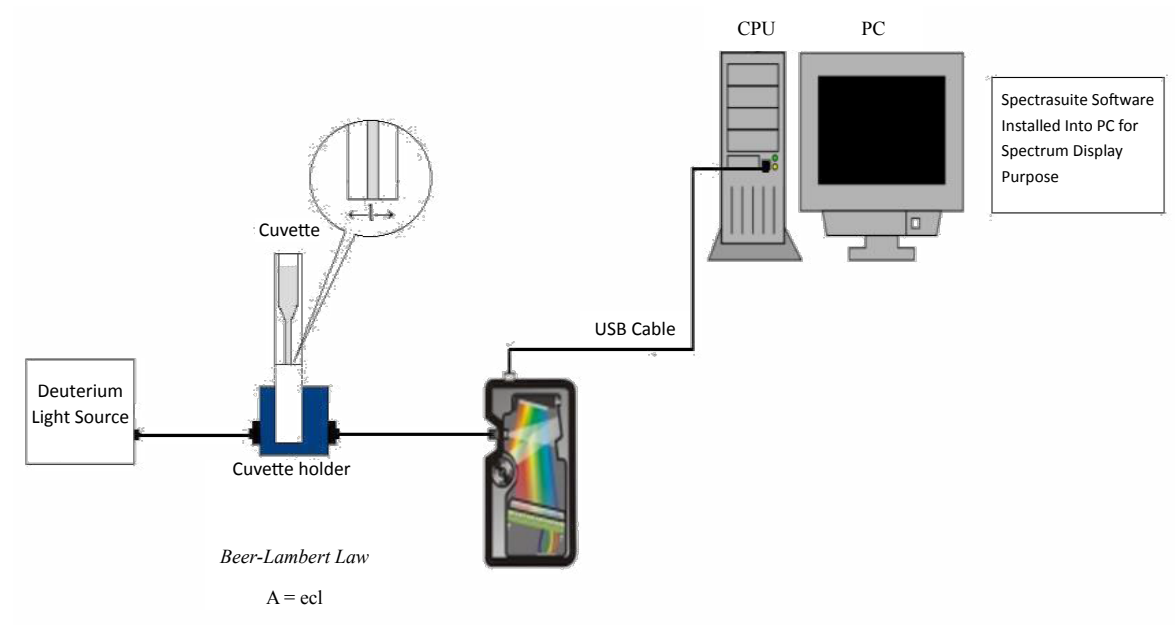

Figure 1: A complete experimental setup for a sample of uric acid in liquid form. 
light absorption of the uric acid compound. The absorption depends on the molecular structure of uric acid as its special characteristics towards the UV light radiation. Uric acid has a particular molar absorptivity or coefficient which may translates its absorption in the UV region. In this research, every concentration of uric acid obtained a different absorbance value at a close or almost a same wavelength. The optimal absorption was obtained at $293.99 \mathrm{~nm}$ which is really close to the values in the previous research studies. The chemical bonding of uric acid also may give an effect to energy excitation and the UV wavelength absorption. High energy is required for a big gap difference of the energy excitation and may result a short wavelength or vice versa. In other words, the chemical bonding of uric acid has its own molecular structure which may give a different energy excitation and the performance on the UV wavelength absorption. In previous research studies, it was reported that uric acid was successfully detected at 293 $\mathrm{nm}, 292 \mathrm{~nm}$ or another close value in the intended UV wavelength region. However, this research proves that pure powder of uric acid with a molecular weight $168.11 \mathrm{~g} / \mathrm{mol}$ still indicated a close value of UV absorption wavelength. Additionally, the spectrometer can measure the different concentrations where the UV wavelength absorption keeps standing on a same wavelength peak. The concentration depends on the amount of uric acid in the sample.

Figure 1 shows the initial resulting curves of the four concentrations of uric acid at a same UV absorption wavelength. The most optimal graph or curve should be selected below 1.0 absorbance to avoid shadows or noise signals that would be created among the molecules or chromospheres if the concentration is really high. The curves of absorbance indicate the concentration values in the intended UV wavelength region, $200 \mathrm{~nm}-400 \mathrm{~nm}$. The highest absorbance was obtained by the uric acid stock, $1 \mathrm{mg} / \mathrm{mL}$ following by the other three concentrations; $0.8 \mathrm{mg} / \mathrm{ml}, 0.6 \mathrm{mg} / \mathrm{mL}$ and $0.4 \mathrm{mg} / \mathrm{mL}$. This experiment was successfully proved that the spectrometer is able to detect the uric acid with grits by different concentrations at a same wavelength peak or band in the UV region. Figure 2 shows the new signals produced using the averaging method (three points interval). They look smoother and lack of noise. The highest wavelength peak is really sharp to indicate the UV absorption belongs to each sample of uric acid (Figures 2 and 3).

\section{Conclusion}

This experiment was aimed to analyze the detection of the uric acid in the UV region. Absorption technique was utilized to investigate where the uric acid absorbs the UV light radiation. The dilutions of the samples were done based on the molarity formula to produce the four different concentrations of uric acid starting from the uric acid stock. The optimal absorption wavelength was obtained at $294.46 \mathrm{~nm}$ by the

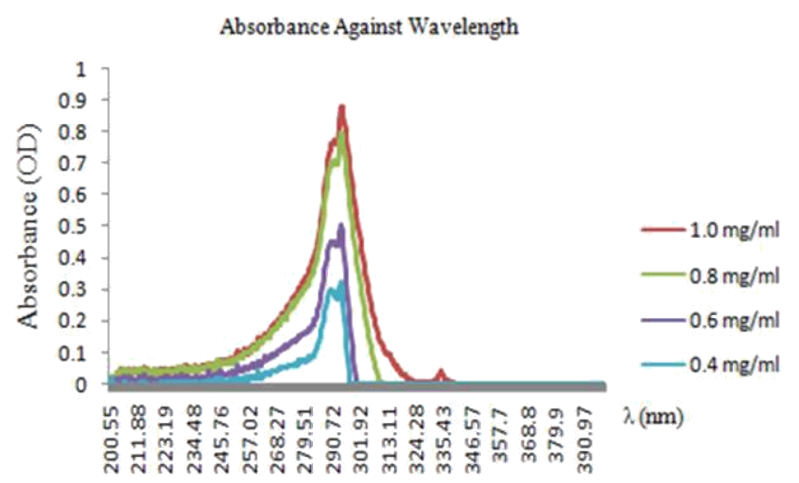

Figure 2: UV absorption wavelength of uric acid with different concentrations.

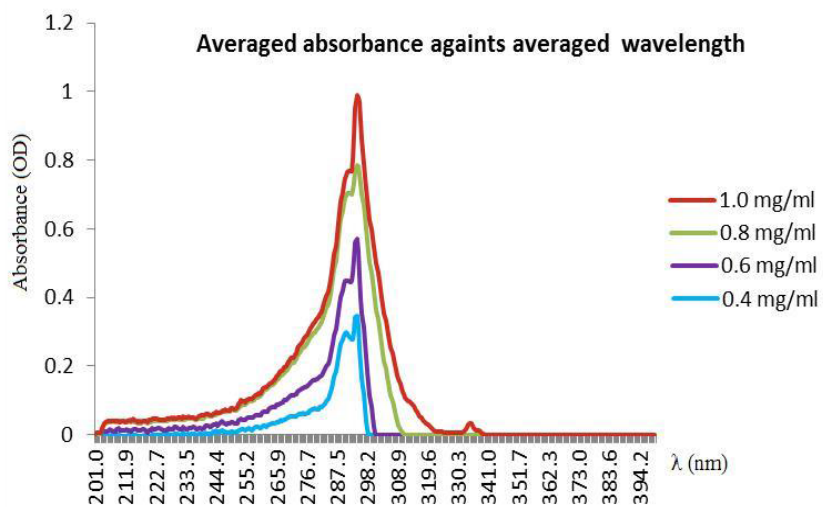

Figure 3: Combination of three wavelengths using averaging method (three points interval).

four different concentrations. Previous research studies showed that the UV absorption wavelength such as $292 \mathrm{~nm}$ and $293 \mathrm{~nm}$ are very close to this value. The four concentrations proportionally indicated different absorbance values in the intended UV wavelength from 200 $\mathrm{nm}$ to $400 \mathrm{~nm}$ at a constant wavelength peak. However, the origin signal was smoothed up to reduce the noise signals and obtain the sharpest absorption wavelength of uric acid at $293.99 \mathrm{~nm}$. Therefore, $293.99 \mathrm{~nm}$ was selected as the best UV absorption wavelength of uric acid. The experiment was proved to give a close value of UV absorption wavelength even with the use of artificial uric acid even with suspended particles. A common practice normally requires a long time or extra structures on the experimental setup for the analysis purposes. The optical sensor is more effective and even may give a fast time response in three seconds. In the future, a research study can be proposed with a sample of human serum as a medical sample.

\section{Acknowledgment}

I would like to thank my supervisor, Dr. Hadi Manap for his full support and guidance. I would also like to thank all the laboratory staffs and science officers to provide me all the tools and instruments for the research use. I really appreciate all the knowledge and guidance as the directions for me complete this research.

\section{References}

1. Ragab G, Elshahaly M, Bardin T (2017) Gout: An old disease in new perspective-A Review. Journal of Advanced Research 8: 495-511.

2. Xu L, Shi Y, Zhuang S, Liu N (2017) Recent advances on uric acid transporters. Oncotarget 8: 100852.

3. Hainer BL, Matheson E, Travis Wilkes R (2014) Diagnosis, treatment, and prevention of gout. American Family Physician 90: 831-836.

4. Idborg H, Zamani L, Edlun PO, Schuppe-Koistinen I, Jacobsson SP (2005) Metabolic fingerprinting of rat urine by LC/MS: Part 1. Analysis by hydrophilic interaction liquid chromatography-electrospray ionization mass spectrometry. Journal of Chromatography B 828: 9-13.

5. Montaseri $\mathrm{H}$, Khajehsharifi $\mathrm{H}$, Yousefinejad $\mathrm{S}$ (2014) UV determination of epinephrine, uric acid, and acetaminophen in pharmaceutical formulations and some human body fluids using multivariate calibration. Química Nova 37: 1404 1409 .

6. Ali SMU, Alvi NH, Ibupoto Z, Nur O, Willander M, Danielsson B (2011) Selective potentiometric determination of uric acid with uricase immobilized on $\mathrm{ZnO}$ nanowires. Sensors and Actuators B: Chemical 152: 241-247.

7. Affum AO (2007) Determination of xanthine and uric acid in xanthinuric urine and extracellular fluid of porcine endothelial cells of the pulmonary artery by high performance liquid chromatography Open Access Theses and Dissertations.

8. Pires NMM, Dong T, Hanke U, Hoivik N (2014) Recent developments in optical detection technologies in lab-on-a-chip devices for biosensing applications Sensors 14: 15458-15479. 
Citation: Norazmi N, Abdul Rasat ZR, Mohamad M, Manap H (2018) UV Detection on Artificial Uric Acid Using UV-Vis Spectrometer. J Laser Opt Photonics 5: 179. doi: 10.4172/2469-410X.1000179

Page 4 of 4

9. Anderson CW (2005) Turbidity Geological Survey Techniques of WaterResources Investigations. USGS 9: 1-55.
10. Kamenev Al, Lyakhov AB (2003) Comparative analysis of mathematical methods for noise filtering in stripping voltammetry. Journal of Analytical Chemistry 58: 257-261 